\title{
Large-amplitude, high-frequency single-molecule switch ${ }^{\dagger}$
}

\author{
Nicolò Ferri ${ }^{1, \#}$, Norah Algethami ${ }^{2, \#}$, Andrea Vezzoli ${ }^{1, \#}$, Sara Sangtarash²,\#, Maeve McLaughlin¹, Hatef Sadeghi², Colin J. \\ Lambert $^{2, *}$, Richard J. Nichols ${ }^{1}$ and Simon J. Higgins ${ }^{1, *}$ \\ 1: Department of Chemistry, University of Liverpool, Crown Street, Liverpool L69 7ZD, U.K. \\ 2: Department of Physics, Lancaster University, Lancaster LA1 4YB, U. K.
}

The design and development of metal/single-molecule/metal junctions with a conductance response to external stimuli has been a strong driving force in molecular electronics community. Reproducible conductance increase (or decrease) of a junction in response to external stimuli have been exploited, for instance, in the development of devices that are sensitive to changes in $\mathrm{pH}^{1,2}$ illumination, ${ }^{3-5}$ specific analytes, ${ }^{6,7}$ and electrochemical/electrostatic potential. ${ }^{8,9}$ Mechanoresistive metal-molecule-metal junctions, whose electrical conductance depends on the mechanical separation of the two electrodes, allow further control, which could be exploited to fabricate junctions responsive to multiple stimuli (e.g. electrochemical potential and electrode separation), for applications as single-molecule Boolean logic gates. ${ }^{10}$ Furthermore, knowledge of the structure-property relationships of mechanosensitive junctions provides a wealth of information about the nature, strength and configuration of metal-molecule interactions at the contact interface, which can be applied to fundamental studies of surface science or organometallic chemistry, and can be exploited to improve the design of molecular devices. We report here metal/single-molecule/metal junctions with high-frequency (10 kHz) mechanoresistive behaviour with enhanced sensitivity, based on (methylthio)thiophene contacting groups. The effect arises from localised interactions between the thienyl sulfurs and the electrodes, which allows the junction to transition from a monodentate to a bidentate contact configuration as the junction is compressed, resulting in a up to $\sim 100 x$ sensitivity boost of the (methylthio)thiophene-terminated molecule, compared with the (methylthio)benzene counterpart.

Changes of electrical conductance associated with the stretching or compression of a single-molecule junction sheds light on force-induced enhancement of molecular transport resonance, ${ }^{11}$ electron transfer reactions, ${ }^{12}$ spin transitions of an organometallic $\mathrm{Fe}(\mathrm{II})$ complex, ${ }^{13}$ stereoelectronic effects ${ }^{14}$ and quantum interference features. ${ }^{15}$ Alterations of the molecule-metal contact configuration ${ }^{16-19}$ upon junction stretching or compression are another important class of phenomena which translates into mechanoresistive behaviour. Enhanced overlap of $\pi$-orbitals with the metallic electrodes in a compressed junction results, for instance, in higher conductance in thiophenol-terminated molecular wires, ${ }^{20}$ or a rheostat-like behaviour in long oligoenes, ${ }^{21}$ demonstrating the importance of weak interactions at the nanoscale. Such interactions are however ill-defined in nature, and to date, the measured conductance changes upon junction size modulation are only moderate. Here we present a study of molecular wires with improved mechanoresistive behaviour, based on a methyl thioether and thienyl S-atom bidentate contact configuration. The former acts as primary contact and grants strong mechanical and electrical coupling, while the latter can interact less strongly with a metallic electrode through the S-atom lone pair and provide the additional electronic coupling to enhance conductance in the compressed junction (Figure 1b). Thiophenes are known to make contact to Au electrodes, ${ }^{22}$ but the interaction is reported as being weaker than traditional contact groups, ${ }^{23}$ thus making it an ideal "supporting" molecular contact to the a

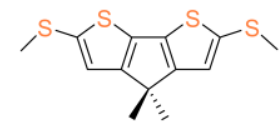

1

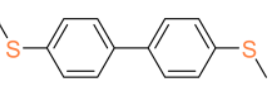

3

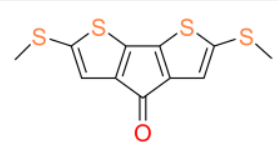

2

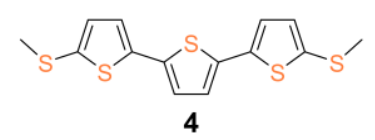

b

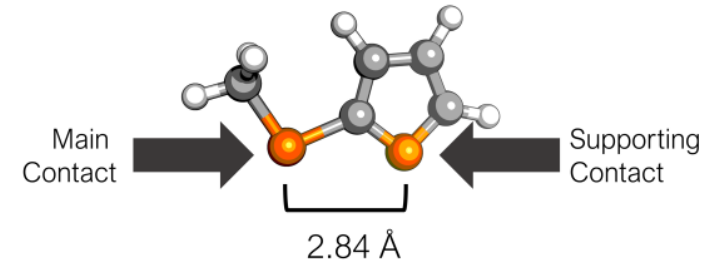

Figure 1: Structures and numbering of the compounds presented in this study (a) and schematics of the designed (methylthio)thiophene contact, with the two binding sites highlighted. Key: $\mathrm{H}=$ white, $\mathrm{C}=$ grey, $\mathrm{S}=$ orange.

stronger methyl thioether. We synthesised the compounds presented in Figure 1a (synthetic details and characterisation in the $\mathrm{SI}$ ), focussing on the bridged bithiophenes $\mathbf{1}$ - 2 to reduce the issues arising from their reduced 5-fold symmetry which results in a large spread of conductance values. ${ }^{24}$

We characterised the electromechanical behaviour of these compounds by employing a modified ${ }^{17,18}$ STM-BJ technique. $^{25}$ In a typical experiment, a piezoelectric actuator drives a $\mathrm{Au}$ tip into a $\mathrm{Au}$ substrate to form a

\footnotetext{
† The supporting information (SI) is available on chemrxiv.org

- Synthetic procedures and characterisation data.

- Further details on the STM-BJ experiments, equipment used, measurement procedure and data analysis algorithms.

- Further theoretical calculations.
}

\#: these authors contributed equally to this work

* Corresponding authors: shiggins@liverpool.ac.uk \& c.lambert@lancaster.ac.uk 

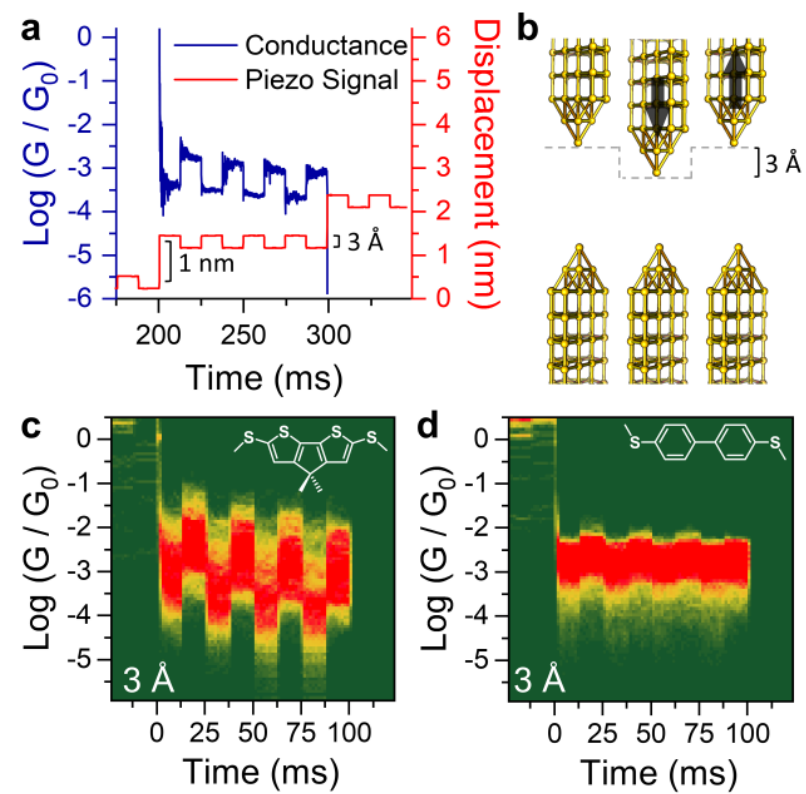

Figure 2: Example of a typical $3 \AA$ modulation measurement (a), with the imposed piezo signal (red) and the corresponding conductance trace (blue), showing the correlation between increase in conductance and junction compression. Idealised position of the electrodes during the modulation is depicted in (b). Conductance vs time density maps for compound $\mathbf{1}$ (c) and 3 (d), under square-wave modulation of $3 \AA$. Density maps are normalised to the number of scans used to compile them, which is 828 (c) and 980 (d). The structure of the molecular wire is superimposed on the relevant density map.

contact having conductance $>5 \mathrm{G}_{0}\left(\mathrm{G}_{0}\right.$ is the quantum of conductance, $\approx 77.48 \mu \mathrm{S})$. The fresh Au-Au junction is then abruptly stretched by $1 \mathrm{~nm}$, and a modulation is applied to the piezo signal for $100 \mathrm{~ms}$. The junction is then further stretched and modulated several times, to ensure the formation of a single-molecule junction, and its subsequent clean rupture, with an overall stretching of 8 $\mathrm{nm}$. The tip is then driven towards the substrate until the contact once again has a conductance $>5 \mathrm{G}_{0}$, and the process is then repeated thousands of times. We then used an algorithm to slice the traces between abrupt stretches and discard the traces with no evidence of junction formation and those where the single-molecule junction did not survive the whole modulation process. On average across the experiment presented here, $38 \%$ of the traces are kept by the algorithm, and these are subsequently compiled in density maps. More details about the measurements and data analysis can be found in the methods section and the SI. We started our investigation on compound 1 by applying a $3 \AA$ squarewave modulation to the piezo actuator, which abruptly changes the electrode separation and holds the position for $12.5 \mathrm{~ms}$ to allow junction relaxation. The amplitude of 3 $\AA$ was chosen as being very close to the S-S distance in the (methylthio)thiophene moiety (Figure 1b).

As can be observed in Figure 2, the square-wave modulation applied to the electrode separation results in large and defined changes in the conductance of $\mathbf{1}$ (Figure 2c). Conductance modulation is reduced when the amplitude of the square wave applied to the piezo is decreased from $3 \AA$ to $2 \AA$ but does not change significantly when the amplitude is increased to $4 \AA$ (See
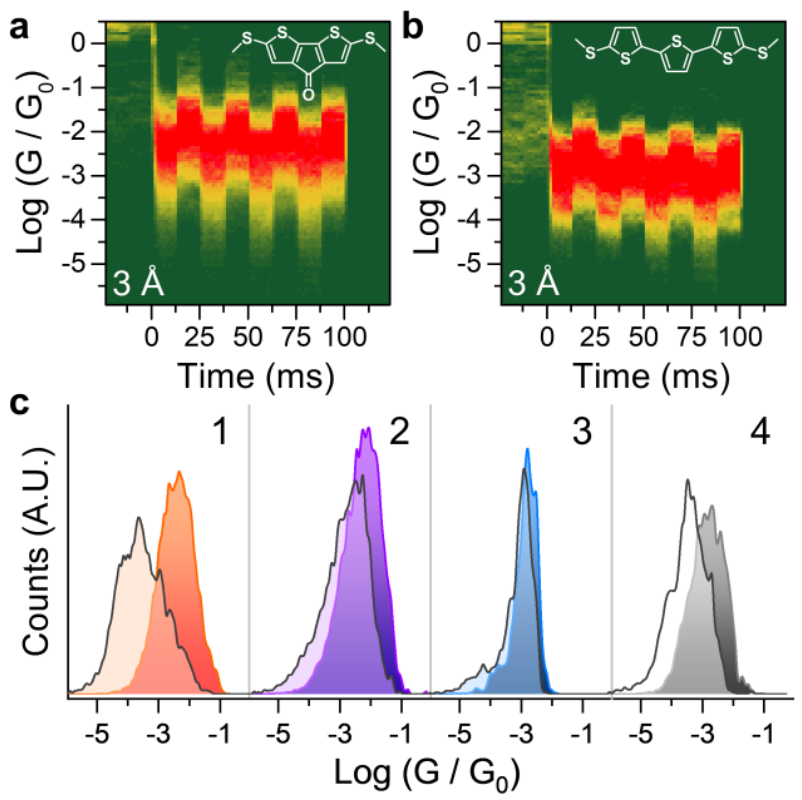

Figure 3: Conductance vs. time density maps of compound 2

(a) and 4 (b) under $3 \AA$ modulation. The structure of the molecular wire is superimposed on the relevant density map. Density maps are normalised to the number of scans used to compile them, which is 1221 (a) and 1072 (b). 1D histograms (c) of the four compounds presented in this study, obtained as profile the density maps at $3 \AA$ modulation. The compressed junction is reproduced in darker colour, and the extended junction is overlaid in lighter colour.

SI for density maps). For comparison, the biphenyl-based compound $\mathbf{3}$ showed a very small conductance change upon modulation (Figure 2d), which might be due to increased electrode-electrode direct tunnelling or the already reported increased interactions of the electrodes with the phenyl $\pi$-system as these junctions are compressed (lateral coupling). ${ }^{20}$ The striking difference in behaviour between these two simple biaryl compounds suggests that the thienyl moiety is responsible for the large mechanoresistive phenomenon. To better characterise this behaviour, we turned our attention to compounds 2 and 4, which would also test the versatility of 2(methylthio)thiophene as a switching contact moiety. In compound 2 the carbonyl substituent has an electron withdrawing effect, and we reasoned this would result in reduced thienyl S-electrode coupling, thereby decreasing the switching magnitude. Compound $\mathbf{4}$ is a longer oligothiophene, and its purpose is to test whether mechanosensitive behaviour is retained in longer molecular wires. By using the same $3 \AA$ square-wave modulation presented earlier we observed conductance changes of significantly reduced magnitude in $\mathbf{2}$ as expected (Figure 3a), and well-defined conductance variations in $\mathbf{4}$ (Figure 3b).

The overall results confirm that the thienyl moiety as responsible for the observed behaviour, with 1 being the compound providing the largest conductance variation upon modulation of the electrode position, as evidenced by analysing the modulation profile (Figure 3c). We therefore exploited this unprecedented high conductance modulation to test the effect of more incremental compression/elongation cycles, and thereby assess the 

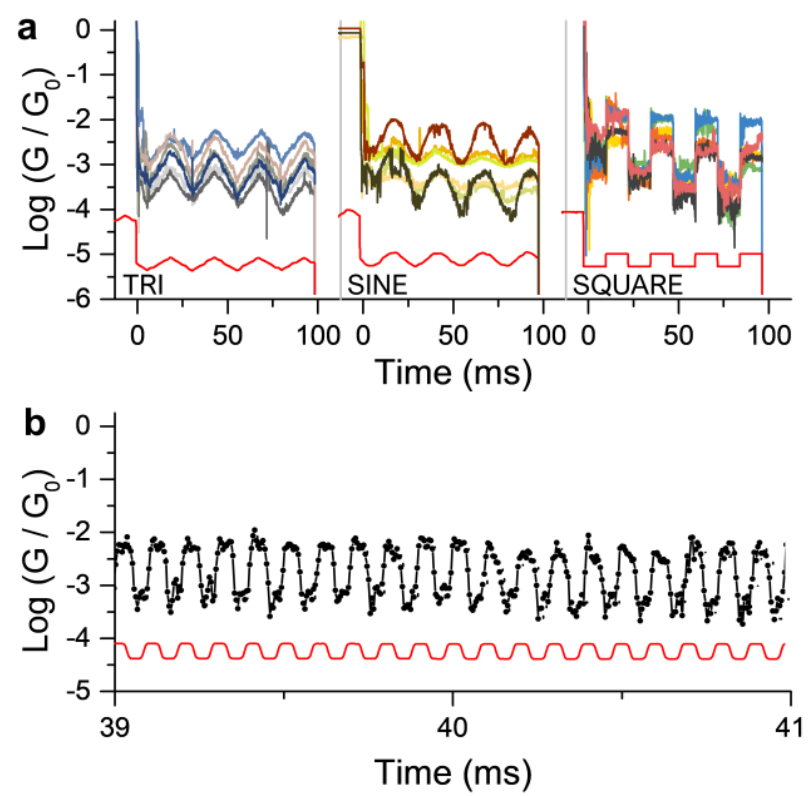

Figure 5: Conductance signal for compound 1 under different types of modulation (a) and under high-speed $(10 \mathrm{kHz})$ trapezoidal modulation (b). The piezo signal is displayed along with the conductance curves in red. Only a 2 ms portion of a single high-frequency modulation trace is displayed here for clarity. Additional data can be found in the SI.

potentiometric behaviour of such single-molecule junctions. Under triangular and sinusoidal modulations, the conductance of 1 consistently follows the signal applied to the piezo actuator, as can be observed in Figure 4a. From the triangular ramp the average dependence of the conductance on electrode separation (the observed slope) was calculated as $e^{0.78 / \AA}$, in good agreement with the data obtained with the square wave modulation, which predicts a dependence of $e^{0.81 / \AA}$. We also performed highfrequency modulation experiments (10 kHz, Figure 4b), and compound 1 showed regular changes in conductance correlated with the signal imposed to the piezo actuator, with no apparent fatigue for up to 1000 modulations (additional details in the SI). It should be noted that a 10 $\mathrm{kHz}$ frequency is at our instrument limit $(200 \mathrm{kSa} / \mathrm{s}$ data acquisition system, resulting in 20 points per modulation cycle) and it is therefore possible that even higher switching frequencies can be attained.

To better understand the phenomena leading to conductance modulation, we used density functional theory (DFT) to compute the conductance versus electrode separation for all the molecules (see SI for details). The quantum transport code Gollum ${ }^{26}$ was used to calculate the transmission coefficient $T(E)$ for electrons of energy $E$ passing from the source to the drain electrode via the molecule. If $T(E)$ varies slowly on the scale of $k_{B} T$ at room temperature $T$, then $G=G_{0} T\left(E_{F}\right)$, where $E_{F}$ is the Fermi energy of the gold electrodes. Using compound 4 as example (Figure 5a), we predict that at small tip-tip distances, the gold electrodes interact with both thienyl and thioether sulfurs, resulting in high molecule-electrode coupling and a high transmission coefficient within the gap between the highest occupied molecular orbital (HOMO) and lowest unoccupied molecular orbital (LUMO) resonances (Figure $5 \mathrm{c}$ ). As the electrode separation is increased, the coupling to the thiophene moiety is reduced a

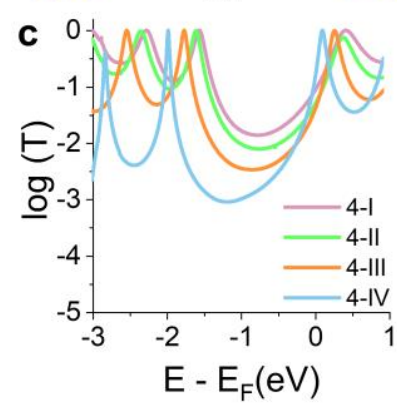

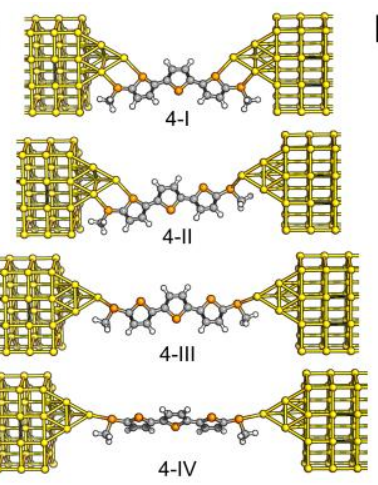

b
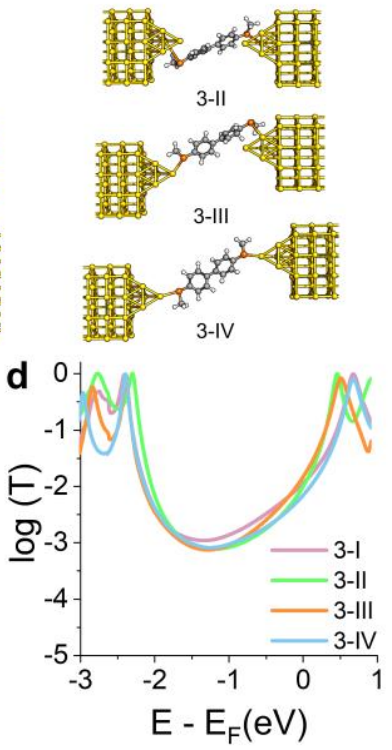
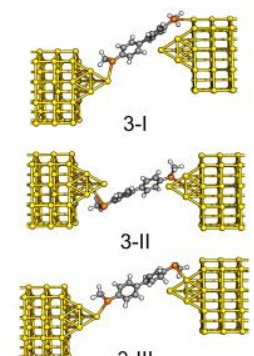

Figure 4: Relaxed structure of molecule 4 (a) and 3 (b) between two Au electrodes, at various tip-tip distances (11 and $8 \AA$ respective starting separation, increased at any step by $2 \AA$ ). Key: $\mathrm{H}=$ white, $\mathrm{C}=$ grey, $\mathrm{S}=$ orange, $\mathrm{Au}=$ yellow.

Corresponding transmission coefficient $T(E)$ versus electron energy of compound $\mathbf{4}$ (c) and $\mathbf{3}$ (d) at the four tip-tip distances. $E$ is plotted relative to the DFT-predicted Fermi energy of gold.

and therefore the value of transmission coefficient decreases.

To predict the effect on the conductance $G=G_{0} T\left(E_{F}\right)$, a value for $E_{F}$ is needed. In Figure 5c-d, the HOMO and LUMO levels correspond to the resonances in the transmission plots $T(E)$ located immediately below and above $E-E_{F}=0$ respectively. The precise values of the HOMO and LUMO levels relative to $E_{F}$ (and therefore the exact zero of the horizontal axis) depend on environmental conditions and on the unknown shape of the electrodes. However, since the molecules are neither oxidised nor reduced, $E_{F}$ lies within the energy gap between the HOMO and the LUMO, and the qualitative change in the conductance with tip-tip distance is determined by the behaviour of $T(E)$ within the gap. In compound 4, the additional thiophene-Au interactions described earlier result in large variations of the mid-gap value of $T(E)$ as the electrode position is modulated, and therefore the conductance is also expected to exhibit large variations. In compound 3, which lacks a thiophene moiety, the value of $T(E)$ within the gap does not change significantly, and the conductance is predicted to be almost independent of the electrode separation. The process was repeated for compounds 1 and $\mathbf{2}$ (details in the SI) and, in agreement with our experiments, compressing the electrodes leads to strong interactions between the electrodes and the thiophene moieties, causing significant variations of the transmission coefficient over a wide range of energies within the HOMO-LUMO gap. Overall the amplitude of transmission coefficient values correlates well with the experimental data, with compound $\mathbf{1}$ having the largest 
variations in $T(E)$ as the electrode separation is modulated, and compound 3 showing little or no effect.

In conclusion, we designed and characterised a series of single-molecule mechanoresistive junctions, based on a bidentate contact moiety that exploits the weak interactions of thienyl sulfurs with $\mathrm{Au}$ electrodes. The junctions displayed high sensitivity to the applied mechanical modulation and can operate at $\mathrm{kHz}$ speed showing no sign of fatigue for $>1000$ cycles. The functional moiety is a (methylthio)thiophene, which is directly responsible for the observed behaviour by providing multiple anchoring points (the thienyl and thioether sulfurs) for the metallic electrodes. Our study presents a novel strategy for the introduction of electromechanical functionality in molecular wires and highlights the importance of weak interactions at the electrode interface. Furthermore, as (methylthio)thiophenes and thiophenethiols are widely used as molecular wire termin in molecular electronics, ${ }^{27-29}$ our results shed more light on their unusual electromechanical properties and provide additional insights for the interpretation of their singlemolecule conductance trajectories during a break-junction experiment.

\section{Methods}

Preparation of compounds 1-4: Compound 1 was synthesised by methylating commercial $4 \mathrm{H}$ cyclopenta[2,1-b:3,4-b']dithiophene with methyl iodide and potassium hydroxide, followed by selective bromination with NBS, halogen-lithium exchange with $n$ butyllithium and quench with dimethyl disulfide. Compound 2 was prepared from commercial 2,2'bithiophene by tetrabromination with elemental bromine, followed by halogen-lithium exchange with $n$-butyllithium and quench with dimethyl disulfide to yield 3,3'-dibromo5,5'-dithiomethyl-2,2'-bithiophene.

Halogen-lithium exchange with $n$-butyllithium, followed by low-temperature quench with dimethylcarbamoyl chloride gave 2 . Compound $\mathbf{3}$ was prepared by halogen-lithium exchange of 4,4'-diiodobiphenyl with $n$-butyllithium, followed by quench with dimethyl disulfide. Compound 4 was prepared by double direct lithiation of $2,2^{\prime}: 5^{\prime}, 2^{\prime \prime}$ terthiophene with $n$-butyllithium, followed by quench with dimethyl disulfide. Detailed procedures and characterisation data can be found in the SI.

Scanning Tunnelling Microscope Break Junction: molecular junctions are formed by repeatedly driving a Au tip into an $\mathrm{Au}$ substrate and then pulling it apart in a solution of the desired molecular wire (mesitylene, $1 \mathrm{mM}$ ). A constant bias $(200 \mathrm{mV})$ is applied between the tip and the substrate during the measurements, which are performed at room temperature. The tip is retracted in a stepwise manner, creating a nanogap which is held for 100 $\mathrm{ms}$ while its size is modulated (see Figure $2 \mathrm{a}$ for the signal applied to the piezoelectric actuator). The tip is then retracted until junction rupture and driven into the substrate again. The process is continuously repeated. 1000 cycles are performed on the bare Au substrate prior to the measurements, to perform a mechanical annealing of the tip and ensure absence of contaminants. After the introduction of the molecular wire, 2000-3000 scans are collected and then analysed using automated algorithms. Details on the instrumentation used and data analysis can be found in the SI.

DFT calculations: the optimized geometry and ground state Hamiltonian and overlap matrix elements of each structure was self-consistently obtained using the SIESTA $^{30}$ implementation of density functional theory (DFT). SIESTA employs norm-conserving pseudopotentials to account for the core electrons and linear combinations of atomic orbitals to construct the valence states. The generalized gradient approximation (GGA) of the exchange and correlation functional is used with the Perdew-Burke-Ernzerhof parameterization (PBE) a double- $\zeta$ polarized (DZP) basis set, a real-space grid defined with an equivalent energy cut-off of 250 Ry. The geometry optimization for each structure is performed to the forces smaller than $10 \mathrm{meV} / \AA$.

Transport calculations: The mean-field Hamiltonian obtained from the converged DFT calculation was combined with Gollum ${ }^{26}$ implementation of the nonequilibrium Green's function method to calculate the phase-coherent, elastic scattering properties of each system consist of left gold (source) and right gold (drain) leads and the scattering region. The transmission coefficient $T(E)$ for electrons of energy $E$ (passing from the source to the drain) is calculated via the relation: $T(E)=$ $\operatorname{Trace}\left(\Gamma_{R}(E) G^{R}(E) \Gamma_{L}(E) G^{R \dagger}(E)\right)$. In this expression, $\Gamma_{L, R}(E)=i\left(\sum_{L, R}(E)-\sum_{L, R}{ }^{\dagger}(E)\right)$ describe the level broadening due to the coupling between left $(L)$ and right $(R)$ electrodes and the central scattering region, $\sum_{L, R}(E)$ are the retarded self-energies associated with this coupling and $G^{R}=\left(E S-H-\sum_{L}-\sum_{R}\right)^{-1}$ is the retarded Green's function, where $H$ is the Hamiltonian and $S$ is overlap matrix. Using obtained transmission coefficient $T(E)$, the conductance could be calculated by Landauer formula $\left(G=G_{0} \int d E T(E)(-\partial f / \partial E)\right)$ where $G_{0}=2 e^{2} / h$ is conductance quantum and $f=1 /[1+$ $\left.\exp \left(E-E_{F}\right) / k_{B} T\right]$, where $E_{F}$ is the Fermi energy. If $T(E)$ veries slowly of the scale of $k_{B} T$ at room temperature $T$, then $G=G_{0} T\left(E_{F}\right)$.

\section{Acknowledgements}

This work was supported by UK EPSRC (grants EP/M005046/1, EP/M029522/1, EP/M014169/1 $E P / P 027156 / 1$, EP/N03337X/1, EP/N017188/1) and the ECH2020 FET Open project 767187 “QuIET”. H.S. and S.S. acknowledge the Leverhulme Trust (Leverhulme Early Career Fellowships no. ECF-2017-186 and ECF2018-375) for funding.

\section{Author Contributions}

A.V., S.J.H. and R.J.N. conceived the project and designed the experiments. N.F, A.V. and M.M. synthesised the compounds used in this study. A.V. developed the STM$B J$ instrument and performed the measurements. A.V., S.J.H. and R.J.N. analysed the experimental data. N.A. and S.S. performed the DFT calculations. S.S., H.S. and C.J.L. developed the theoretical explanation. A.V., S.S. and 
C.J.L. wrote the manuscript. All authors discussed the results and commented on the manuscript.

\section{Data Availability}

NMR spectra (Bruker format) for compounds $\mathbf{1 - 4}$ and raw STM-BJ data can be found in the data catalogue in Liverpool at https://datacat.liverpool.ac.uk/id/eprint/549 and at DOI: 10.17638/datacat.liverpool.ac.uk/549.

\section{References}

1. Li, L., Lo, W.-Y., Cai, Z., Zhang, N. \& Yu, L. Protontriggered switch based on a molecular transistor with edge-on gate. Chem. Sci. 7, 3137-3141 (2016).

2. Brooke, R. J. et al. Dual Control of Molecular Conductance through $\mathrm{pH}$ and Potential in SingleMolecule Devices. Nano Lett. 18, 1317-1322 (2018).

3. Zhou, J., Wang, K., Xu, B. \& Dubi, Y. Photoconductance from Exciton Binding in Molecular Junctions. J. Am. Chem. Soc. 140, 7073 (2018).

4. Fung, E. D., Adak, O., Lovat, G., Scarabelli, D. \& Venkataraman, L. Too Hot for Photon-Assisted Transport: Hot-Electrons Dominate Conductance Enhancement in Illuminated Single-Molecule Junctions. Nano Lett. 17, 1255-1261 (2017).

5. Jia, C. et al. Covalently bonded single-molecule junctions with stable and reversible photoswitched conductivity. Science 352, 1443-1445 (2016).

6. Vezzoli, A. et al. Gating of single molecule junction conductance by charge transfer complex formation. Nanoscale 7, 18949-18955 (2015).

7. Nishino, T., Shiigi, H., Kiguchi, M. \& Nagaoka, T. Specific single-molecule detection of glucose in a supramolecularly designed tunnel junction. Chem. Commun. 53, 5212-5215 (2017).

8. Pobelov, I. V., Li, Z. \& Wandlowski, T. Electrolyte Gating in Redox-Active Tunneling Junctions: An Electrochemical STM Approach. J. Am. Chem. Soc. 130, 16045-16054 (2008).

9. Brooke, R. J. et al. Single-Molecule Electrochemical Transistor Utilizing a NickelPyridyl Spinterface. Nano Lett. 15, 275-280 (2015).

10. Meng, F. et al. Orthogonally modulated molecular transport junctions for resettable electronic logic gates. Nat. Commun. 5, 3023 (2014).

11. Bruot, C., Hihath, J. \& Tao, N. Mechanically controlled molecular orbital alignment in single molecule junctions. Nat. Nanotechnol. 7, 35-40 (2011).

12. $\mathrm{Li}, \mathrm{Y}$. et al. Mechanical Stretching-Induced Electron-Transfer Reactions and Conductance Switching in Single Molecules. J. Am. Chem. Soc. 139, 14699-14706 (2017).
13. Frisenda, R. et al. Stretching-Induced Conductance Increase in a Spin-Crossover Molecule. Nano Lett. 16, 4733-4737 (2016).

14. Su, T. a., Li, H., Steigerwald, M. L., Venkataraman, L. \& Nuckolls, C. Stereoelectronic switching in single-molecule junctions. Nat. Chem. 7, 1-6 (2015).

15. Stefani, D. et al. Large Conductance Variations in a Mechanosensitive Single-Molecule Junction. Nano Lett. 18, 5981-5988 (2018).

16. Yoshida, K. et al. Correlation of breaking forces, conductances and geometries of molecular junctions. Sci. Rep. 5, 9002 (2015).

17. Quek, S. Y. et al. Mechanically controlled binary conductance switching of a single-molecule junction. Nat. Nanotechnol. 4, 230-234 (2009).

18. Ismael, A. K. et al. Side-Group-Mediated Mechanical Conductance Switching in Molecular Junctions. Angew. Chemie Int. Ed. 56, 1537815382 (2017).

19. Ramachandran, R. et al. An Electromechanical Approach to Understanding Binding Configurations in Single-Molecule Devices. Nano Lett. 18, 6638-6644 (2018).

20. Diez-Perez, I. et al. Controlling single-molecule conductance through lateral coupling of $\pi$ orbitals. Nat. Nanotechnol. 6, 226-231 (2011).

21. Meisner, J. S. et al. A single-molecule potentiometer. Nano Lett. 11, 1575-1579 (2011).

22. Harzmann, G. D., Frisenda, R., van der Zant, H. S. J. \& Mayor, M. Single-Molecule Spin Switch Based on Voltage-Triggered Distortion of the Coordination Sphere. Angew. Chemie Int. Ed. 54, 13425-13430 (2015).

23. Bejarano, F. et al. Robust Organic Radical Molecular Junctions Using Acetylene Terminated Groups for C-Au Bond Formation. J. Am. Chem. Soc. 140, 1691-1696 (2018).

24. Dell, E. J. et al. Impact of molecular symmetry on single-molecule conductance. J. Am. Chem. Soc. 135, 11724-7 (2013).

25. Xu, B. \& Tao, N. Measurement of Single-Molecule Resistance by Repeated Formation of Molecular Junctions. Science 301, 1221-1223 (2003).

26. Ferrer, J. et al. GOLLUM: a next-generation simulation tool for electron, thermal and spin transport. New J. Phys. 16, 093029 (2014).

27. Zang, Y. et al. Resonant Transport in Single Diketopyrrolopyrrole Junctions. J. Am. Chem. Soc. 140, 13167-13170 (2018).

28. Cai, Z. et al. Exceptional Single-Molecule Transport Properties of Ladder-Type Heteroacene Molecular Wires. J. Am. Chem. Soc. 138, 10630-10635 (2016). 
29. Dulić, D. et al. One-Way Optoelectronic Switching of Photochromic Molecules on Gold. Phys. Rev. Lett. 91, 207402 (2003).

30. Soler, J. M. et al. The SIESTA method for ab initio order- N materials simulation. J. Phys. Condens. Matter 14, 2745-2779 (2002). 